\title{
Nursing students' perceptions of obesity and behaviour change: Implications for undergraduate nurse education
}

\author{
Chris Keyworth ${ }^{\mathrm{a}, *}$, Sarah Peters ${ }^{\mathrm{b}}$, Anna Chisholm ${ }^{\mathrm{b}}$, Jo Hart ${ }^{\mathrm{c}}$ \\ a School of Community Based Medicine, University of Manchester, Manchester, UK \\ b School of Psychological Sciences, University of Manchester, Manchester, UK \\ c School of Medicine, University of Manchester, Manchester, UK
}

\section{A R T I C L E I N F O}

\section{Article history:}

Accepted 16 May 2012

\section{Keywords:}

Nursing students

Qualitative

Overweight

Obesity

Interviews

Behaviour change

Weight management

\section{S U M M A R Y}

Background: Rates of obesity are rising and previous research suggests this is not effectively dealt with in healthcare settings. Nurses are increasingly involved in lifestyle management of patients, and understanding the barriers to discussing weight with patients is likely to increase successful weight management. Obesity management is a role that nursing students will need to be equipped with and more likely to be targeted for future training developments in tackling the increasing rates of obesity.

Objectives: To explore the perceptions of obesity, potential barriers to successful patient weight management and training needs of nursing students.

Methods: Qualitative, semi-structured interviews were conducted with 20 nursing students. Audiotaped interviews were transcribed verbatim and analysed using an inductive thematic approach informed by principles of grounded theory.

Results: Participants reported the challenge of managing obesity in healthcare practice, such as the impact of negative attitudes in healthcare practice on patient care. Although perceived as core to their training, nursing students lacked the confidence and techniques to discuss weight management with patients. Participants also perceived the nursing curriculum as lacking a focus on obesity, and reported a need for advanced communication skills training.

Conclusion: Although seen as important, nurses lack the skills to facilitate weight management, leading to nurses failing to broach the issue.

Practice implications: Nurse educators should consider the perceptions of current students when making curriculum developments in this area.

(c) 2012 Elsevier Ltd. All rights reserved.

\section{Introduction}

Obesity is a worldwide challenge to public health, affecting virtually all ages and socio-economic groups in both developed and developing countries (World Health Organisation, 2000). Approximately 1.1 billion people in the world are overweight (body mass index (BMI) $>25 \mathrm{~kg} / \mathrm{m}^{2}$ ), of which 312 million are obese (BMI $>30 \mathrm{~kg} / \mathrm{m}^{2}$ ) (Haslam and James, 2005), bringing an increased risk of a number of diseases such as diabetes, coronary heart disease, stroke, cancer, and osteoarthritis (Wang et al., 2011). There are also major cost implications associated with treating obesity-related diseases, for example in the United Kingdom, where the number of obese people has nearly trebled in the last 20 years (The

\footnotetext{
* Corresponding author at: School of Community Based Medicine, Williamson Building, University of Manchester, Oxford Road, Manchester, M13 9PL, UK. Tel.: +44 161306 7949.

E-mail address: christopher.keyworth@manchester.ac.uk (C. Keyworth).
}

Health and Social Care Information Centre, 2011), with treatment costs at an estimated $£ 45.5$ billion per year (King, 2011).

Healthcare professionals have a responsibility not only to provide care and promote behaviour change in overweight and obese patients, but to also implement weight loss interventions to increase activity and diet (National Institute for Health and Clinical Excellence, 2006). In particular nurses have an increasing role in providing lifestyle interventions to facilitate weight management (United States Department of Health and Human Services, 2001) such as supporting patients to achieve weight loss goals and to maintain the necessary behavioural changes over time (Lang and Froelicher, 2006). Primary care settings provide an excellent opportunity for healthcare professionals to raise the issue of being overweight, and to begin the process of weight loss (Michie, 2007). Nurses are increasingly working in primary care with responsibility for management of long-term conditions, such as diabetes, where lifestyle change is a key component (Department of Health, 2002). Further, nurses are also becoming increasingly involved in lifestyle management of patients, such as smoking cessation (World Health Organisation, 2003). Research suggests behaviour change techniques can be effectively used by healthcare professionals (Abraham 
and Michie, 2008; Michie et al., 2011), and could be used within consultations where discussions with patients focus upon behaviour change.

Previous research suggests that general practitioners (GPs) do not take opportunities to discuss weight with patients, often due to time constraints (Bocquier et al., 2005). Further, the GP's role has previously been described as involving addressing the primary medical issue a patient attends with (such as diabetes or high blood pressure), consequently referring overweight patients to another healthcare professional to manage their health behaviours (Timmerman et al., 2000). Nurses are especially well positioned to provide important educational information, such as recommended treatment guidelines, as well as encouraging and supporting patients to achieve weight loss (Lang and Froelicher, 2006). Thus, it could be argued that nurses are in a key position to facilitate behaviour change management.

Despite widespread recognition regarding the role for nurses in supporting obese patients to change unhealthy behaviours, a growing body of research suggests there may be barriers that prevent this from occurring in medical practice. These include a lack of training to motivate patients to change their behaviour and the perceived non-compliance of patients to engage in behaviour change communication (Nawaz and Katz, 2001). The presence of such barriers may have an impact on facilitating behaviour change, specifically the implementation of psychological interventions such as opportunistic health promotion (Brown, 2006).

Whilst there is a growing body of evidence exploring practice nurses perceptions of obesity and barriers to patient weight management, little is known about the perceptions amongst trainee nurse samples. As nurse education is known to affect the quality of future health care practice (Poon and Tarrant, 2009), it is necessary that research is conducted to inform the development of nursing practice in tackling obesity. Further, research suggests that most attitudes and skills do not develop with age and experience; rather they are developed at undergraduate level (Dunn and Hansford, 1997). Thus, targeting students may be crucial in informing future nursing curriculum developments.

This study aimed to explore (1) nursing students' perceptions of obesity; and (2) the potential barriers to successful patient weight management.

\section{Methods}

\section{Study Sample and Design}

Participants were recruited from the undergraduate nursing course at a large university in the Northwest of England. The authors approached course administrators who facilitated recruitment emails to be sent to nursing students and posters to be displayed around the university campus. A purposive sampling method (Pope et al., 2002) was used to recruit nursing students to ensure diversity in age, sex and field of nurse training (adult, child and mental health), and thus seeking to capture the widest variation of existing views in order to explore the research question. Recruitment followed principles of constant comparative methodology (Strauss and Corbin, 1998) and was ongoing until thematic saturation was achieved, the point at which gathering data no longer revealed new properties of the core theoretical themes (Charmaz, 2006).

\section{Data Collection}

Digitally audio-recorded semi-structured individual interviews were conducted by one of the authors (CK). A flexible topic guide containing a series of open-ended questions was used to explore a number of topics around nursing students' perceptions about obesity and barriers to weight management (see Box 1). The interviewer combined openended questions to elicit free responses with more focused probing

\section{Box 1}

Areas covered in the topic guide.

- Experience of overweight and obese patients.

- Perceptions of the causes and management of obesity.

- Expectations of their role as a nurse, and other health professionals in managing patients' weight.

- Experience of training in obesity and weight management.

- Experience and understanding of behaviour change interventions.

- Experience and perceptions of current communication skills training.

questions, to encourage unanticipated statements and stories to emerge (Pope et al., 2002).

The order and exact content of the questions were adapted according to each participant's responses and also informed by the ongoing analysis. By modifying the topic guide researchers explored emerging themes in subsequent interviews and follow ideas that arose directly from incoming data.

\section{Ethical Considerations}

All participants provided written consent prior to participation in the interviews and the study was approved by a university ethics committee (reference 517/07P). Any identifying information within the interview (e.g. patient or healthcare professional names/locations) was removed from the audio recording transcripts. All participants were assured that participation was voluntary and they had the right to withdraw without being penalised. The transcribed files were stored in a secure folder on a university computer system.

\section{Analysis}

With the absence of any prior theoretical framework, an inductive thematic approach to the analysis was informed by the principles of Grounded Theory (GT). This iterative process allowed for emerging ideas to be tested in subsequent interviews. Thus, constructive questions were formulated and themes were not sought out, but allowed to emerge from the data (Lyons and Coyle, 2007). By adhering to these principles, the authors were able to control the research process and increase the analytical power of the findings, by developing the analytical framework that was used to view and synthesise the data (Charmaz, 2006).

Coding was conducted by one of the authors (CK), and themes were discussed and agreed upon with the co-authors (SP, JH and AC) of the study during ongoing research meetings. In accordance with Strauss and Corbin's (1998) approach to GT, coding began soon after the first interview was completed. As the interviews progressed and a list of codes was developed, possible themes and sub-themes were developed (Strauss and Corbin, 1998). A process consisting of open coping, axial coding and selective coding was adopted. Open coding allowed the data to be examined in order to expose categories of information. Axial coding aims to "identify a single category as the central phenomenon" (Creswell, 1998, p.151), where selective coding creates a narrative based on the themes previously identified, creating links between themes and sub-themes (Creswell, 1998). The data were examined in detail, and themes were developed whilst keeping in mind the inductive aims of the study and ensuring the data developed further insight (Charmaz, 2006). Where no new information arose from the data, sampling, data collection and analysis ceased (Strauss and Corbin, 1998). 
Rigour

To reinforce the credibility of the analysis, themes were identified and formulated during ongoing research meetings with all authors until a consensus was reached. To ensure transparency and to illuminate the reasoning underpinning the analysis, the complete procedure was documented (Muldoon et al., 2007). In addition, field notes were maintained (Koch, 1994), and peer debriefing conducted (Mariano, 1995), where the researcher conducting the analysis was questioned by other members of the research team, ensuring the interpretations of the data were consistent.

To maximise trustworthiness of the data, the process of researcher triangulation was used. That is, using a range of perspectives from the research team to discuss and interpret the data (Peters, 2010). A peerchecker, a colleague experienced in qualitative methodology, was used to confirm the themes found in the data, and to further increase trustworthiness of the emerging analysis (Strauber and Carpenter, 2003).

\section{Results}

The final sample $(n=20)$ included 19 female participants and 1 male participant, with variations in age (range $=20-40$ years old, median $=24.45$ ) and branches of nurse training (child, $n=4$; adult, $n=14$; and mental health, $n=2$ ). Participants' ethnic backgrounds were White British, $(n=17)$, Asian British $(n=2)$, and Indian $(n=1)$.

Two major themes that describe nursing students' perceptions of obesity and the potential barriers to successful patient weight management were identified: (1) challenges faced when managing patients and (2) perceptions of current training. Illustrative quotes are provided verbatim to illustrate themes, with participant ID number, field and year of training displayed in parentheses.

\section{Challenges Faced When Managing Patients}

Students perceived a number of challenges surrounding managing obesity in healthcare practice: conflicting views over whose role it is to manage weight loss, negative attitudes of nurses, and the influence of nurses' own weight on the ability to elicit change in patients.

\section{Role Responsibility}

Participants displayed contradictions in describing whose role it should be to discuss weight loss plans with patients. A number of different views regarding responsibility of weight management were given, such as the importance of a multi-disciplinary approach from healthcare professionals. On the other hand, some believed that the responsibility lies with the GP, for example as a justification of higher salary:

'Ideally the GP 'cause he gets paid more. He's getting paid to have those people on his books and to do various tests and things...not that I'm saying the nurse isn't skilled and she wouldn't want that responsibility but that patient belongs to that surgery not to the nurses.' (04; Adult; 3rd year)

Some participants believed however, that the responsibility of weight management lies with the patient, as patients receive the relevant information and are expected to take control over their own obesity-related behaviours. However, it was perceived that patients did not always agree that it was their own responsibility to change, which was seen as problematic for nurses. Further to this, it was believed that some patients expected the nurse to take responsibility about their weight; an expectation that participants viewed as unrealistic:

'I think it's more patient triggered at the moment...I don't think it's us making that first step, I think it's waiting for the patient to say 'I want to do something about it.' (19; Adult; 2nd year)
In contrast, other participants welcomed a role of helping patients achieve weight loss goals, and believed that they have a responsibility to broach the subject to fulfil their 'duty of care'. This was supported with the belief that they are well placed to address weight management:

'It's the duty of the health professional, its their obligation to educate the patient as much as they can by stating what the problem is and making them aware of the problem and the consequences, and then finding interventions to help them.' (13; Adult; 2nd year)

\section{Witnessing Negative Attitudes Amongst Qualified Nurses}

Whilst a potential role in helping patients achieve weight loss goals was identified, participants also reported witnessing negative attitudes in other nurses in healthcare practice, such as concerns surrounding the practical implications of managing participants. This resulted in discriminative beliefs towards patients, with participants being aware of the negative impact this would have on patients' experience of healthcare settings:

'They [nurses] were quite discriminative about it, how they sort of took the mick out of the patient...it was a bit kind of eye-opening to what that kind of setting can be like, but yeah I felt sorry for the patient 'cause he was being judged on his appearance....all the way through his A\&E experience he was being mocked a little bit.' (07; Adult; 3rd year)

Some also expressed judgemental attitudes towards patients. Although participants were aware of the negative impact pejorative beliefs could have on patients' experience of healthcare settings, participants blamed the patient for being overweight due to a lack of self-control and failing to adopt a healthy lifestyle, leading to negative perceptions of obese patients:

'You do just immediately think how have you let yourself get that way... it's not very pleasant to look at.' (03; Adult; 3rd year)

\section{Influence of Nurses' Weight}

Further, participants believed that the contribution of a nurse's own weight may affect communication of weight management strategies. Participants expressed that some nurses do not act as appropriate role models by being overweight and that this negatively affects their credibility as weight management advisors:

'I've just found the stereotypical version of a nurse, you know quite big even though they are on the go. So I think for a nurse maybe who is a bit overweight to talk to a patient about it [weight management] just doesn't seem right.' (03; Adult; 3rd year).

'On one of my last wards my mentor was a very large nurse and she wouldn't even bring up the subject of weight because she felt that she had no right to.' (14; Adult; 3rd year)

\section{Perceptions of Current Training}

\section{Lack of Focus on Obesity}

Participants reported concerns about the paucity of training in weight management facilitation. Participants believed that current training was too general and lacked focus about how to manage overweight patients specifically. Where training did focus on health behaviours, these were typically smoking and alcohol use rather than diet and exercise. Furthermore, training focused more upon the reasons why individuals behave unhealthily, rather than providing techniques for facilitating behaviour change.

'There's alcohol and smoking more, we had some psychology lectures of why do people act in certain ways, why do they choose these behaviours, but not so much how to stop them from doing it.' (06; Adult; 3rd year) 
'I don't think in the university you get much training about it to facilitate it [weight loss].' (10, Adult; 3rd year)

\section{Lack of Exposure to Overweight Patients}

Participants reported that their nursing placements were reflective of the perceived emphasis placed on other health behaviours in the curriculum such as smoking and alcohol use. Participants reported having a lack of contact with overweight and obese patients during placements which reinforced their perception that weight management was not a priority condition to address, compared to other health related behaviours. Thus, participants believed that they were unaware of interventions to change people's health behaviours, due to a lack of experience of these through their work placements.

'There's like alcohol use is quite big at the moment I've noticed in my last two placements.' (10; Adult; 3rd year)

'I haven't had much experience of [obese] patients either...if I had been on the right sort of placement and that placement focused on that I would have been fine.' (04; Adult; 3rd year)

\section{Current Level of Communication Skills}

Whilst participants acknowledged that communication skills training was a central part of the nursing curriculum, discussing weight management in particular was perceived as a difficult task, due to the perceived sensitivity of the issue. Therefore discussing weight management with patients was perceived as a specialist skill. As a consequence, participants were unable to relate their general communication skills training to discuss weight management with patients

'We get a lot of communication skills, but we don't get communication taught to us in how to deal with sensitive issues so much.' (12; Adult; 3rd year)

The perceived lack of specialised communication skills for discussing weight with patients also led to the belief that engaging in such discussions might damage the nurse-patient relationship. A number of views were expressed, such as fear of offending the patient (or in the case of overweight children, not wanting to place blame on the parents), and thoughts of not only embarrassing the patient, but also themselves. It was therefore reported that participants might avoid discussing the subject of weight with their patients:

'For you to identify it and then for you to broach the subject is embarrassing for a nurse to go up and say would you like any help. There's probably a great fear of insulting that person, breaking down a trusting relationship.' (16; Adult; 2nd year)

\section{Sceptical of Current Weight Loss Interventions}

Further, participants expressed having limited knowledge and education regarding weight management and wanted to know more about resources available to them. This training need was associated with additional training in specific weight management strategies and in identifying elements of the role that the healthcare professional can play in managing patients' weight. Participants were sceptical of current educational interventions, such as giving advice, or providing information leaflets:

'They might have already heard all this before, and they might think that you're lecturing them...it's not always helpful is it.' (02; Child; 3rd year)

'You can give people advice as much as you want, give them leaflets and hand them things that you think are gonna be really useful, it won't impact on them in any way or they'll think okay for five minutes then we'll walk away and they'll put the leaflet in the bin.' (11; Adult; 3rd year)

\section{Discussion}

This study provides insights into a limited evidence base of the perceptions of nursing students in discussing weight management with patients, and their undergraduate education. We identify a number of key issues surrounding how nursing students communicate with patients and their perceived role in managing obesity, and how this may affect their future practice. Adding to previous research which has demonstrated that qualified nurses hold negative judgements towards obese patients (Brown, 2006), our study demonstrated that some nursing students have already developed negative views of these patients. Further, they reported observing that qualified nurses held negative judgements about obese patients and that discussing obesity could be difficult due to concerns around being able communicate with patients about this. Whilst some participants welcomed a role in helping patients to achieve long-term weight loss goals, they also avoided discussing weight management to prevent offending or embarrassing patients. Participants reported that this may be especially difficult if nurses themselves were overweight and that parent attitudes to unhealthy diets also could be a particular barrier to discussing obesity. Due to such barriers, the patients' health needs were not being addressed.

In relation to nursing students' professional development, being able to discuss weight management with patients whilst being aware that this may be a sensitive issue is a skill that students must develop in order to provide effective care for overweight and obese patients consistent with NICE (2006) guidelines. For example, the inability to communicate with patients due to the presence of negative attitudes towards the care of obese patients may not only affect the quality of care provided (Petrich, 2000; Huizinga et al., 2010), but may also weaken the provider-patient relationship (Harvey and Hill, 2001), and result in obese individuals failing to seek health care (Brownell and Puhl, 2003).

Whilst previous research shows that healthcare professionals believe patients to be personally responsible for their weight (Harvey and Hill, 2001), the present study suggests that the picture may be more complex. Whilst some participants believed that obesity is due to a lack of will power concerning food and thus suggested patients are unmotivated to change, others were unsure of where the responsibility of weight management lies. Previous studies with GPs suggest they are also unclear where the responsibility lies, and fear that discussing weight issues is detrimental to the doctor-patient relationship (Epstein and Ogden, 2005). In the present study however, some nursing students believed the responsibility does lie with the GP, for example as a justification of higher salary. Thus, it remains unclear who should adopt responsibility for weight management, as research suggests doctors and medical students also feel illequipped to facilitate health behaviour change (Chisholm et al., 2012-a).

The present study suggests that nursing students not only believed that broaching weight management may be detrimental to the nursepatient relationship; they were also unaware of how weight management might be managed within a team. Clarifying the roles and responsibilities of nurses during students' early training may help to alleviate the confusion evident in our study, and may result in the successful delivery of health behaviour change interventions (Taylor et al., 2010).

\section{Limitations}

This study is based on participant perceptions through self-report and further research should aim to build on the issues raised in this paper through examining actual behaviour during interactions with patients. It is also acknowledged that due to the study being conducted in only one locality and having a limited range of participants of non-white ethnicity, it may not be possible to generalise these findings to the wider population of nursing students. Cultural 
differences may also warrant further investigation, as past research has shown for instance that nurses from different cultures report different healthcare experiences (Watson et al., 2003). Conclusions drawn from the present findings cannot therefore be generalised beyond a White British population.

\section{Conclusion}

The present study illustrated that whilst nursing students were aware of their potential role in facilitating behaviour change, the perceived challenges in healthcare practice specifically for overweight and obese patients, and a perceived lack of obesity-related communication training were perceived as substantial barriers to effective behavioural management. Current communication training may therefore be improved by focusing upon overcoming the barriers identified in our study, to equip healthcare practitioners to facilitate behaviour change. Nursing students perceived themselves as being ill-equipped with the skills to communicate effectively with patients about weight management, and consequently may avoid the issue. Future research should seek to clarify the extent to which these attitudes and beliefs are shared amongst all nursing specialisms and beyond training, as the barriers to effective patient management found in our study may hinder the implementation of future weight loss interventions in primary care.

This study suggests that nursing students perceive a need for more information regarding obesity and weight management, combined with training in complex communication skills to discuss weight with patients, and provide more effective support to help patients maintain sustained behaviour change. This may also serve to change the pessimistic attitudes towards patients' ability to lose weight. The evidence base of how best to deliver this type of training is limited. A recent systematic review of lifestyle change education interventions for medical students around weight management revealed evidence that was highly susceptible to bias and largely atheoretical (Chisholm et al., 2012-b). Further work is needed to examine a similar evidence base for nurse training and establish what technique can be usefully taught to nurses and nurse trainees.

Evidence from undergraduate medical education suggests that small group experiential communication training is effective in improving skills in health professional trainees (von Fragstein et al., 2008). In addition, the authors argue that discussion of attitudes by health professionals and others in this area could be covered through ethics components of nursing education.

Understanding more about nursing students' attitudes towards the management of obese patients may guide the engagement of students in more effective undergraduate weight management training. Insights gained from our study can inform nurse educators and curriculum developers on the inclusion of more specific obesity-related training as part of undergraduate programmes.

\section{Acknowledgements}

The authors would like to thank all of the participants involved in this study.

Conflict of Interest: No conflict of interest has been declared by the authors.

Funding: This study was partly funded by a British Psychological Society Division of Health Psychology research internship award (Keyworth_BPSDHP2010).

\section{References}

Abraham, C., Michie, S., 2008. A taxonomy of behavior change techniques used in interventions. Health Psychology 27, 379-387.

Bocquier, A., Verger, P., Basdevant, A., Andreotti, G., Baretge, J., Villani, P., Paraponaris, A., 2005. Overweight and obesity: knowledge, attitudes, and practices of general practitioners in France. Obesity Research 13, 787-795.
Brown, I., 2006. Nurses' attitudes towards adult patients who are obese: literature review. Journal of Advanced Nursing 53 (2), 221-232.

Brownell, K., Puhl, R., 2003. Stigma and discrimination in weight management and obesity. The Permanente Journal 7 (3), 21-23.

Charmaz, K., 2006. Constructing grounded theory: a practical guide through qualitative analysis. Sage, Beverly Hills, CA.

Chisholm, A., Hart, J., Lam, V., Peters, S., 2012a. Current challenges of behavior change talk for medical professionals and trainees. Patient Education and Counselling 87 (3), 389-394.

Chisholm, A., Hart, J., Mann, K., Harkness, E., Peters, S., 2012b. Preparing medical students to facilitate lifestyle changes with obese patients: a systematic review of the literature. Academic Medicine. 87 (7) Advance online publication. Retrieved from http://www. ncbi.nlm.nih.gov/pubmed/22622210.

Creswell, J., 1998. Qualitative inquiry and research design: choosing among five traditions. Sage, Thousand Oaks, CA

Department of Health, 2002. Liberating the talents: helping primary care trusts and nurses to deliver the NHS plan. Department of Health, London.

Dunn, S.V., Hansford, B., 1997. Undergraduate nursing students' perceptions of their clinical learning environment. Journal of Advanced Nursing 25 (6), 1299-1306.

Epstein, L., Ogden, J., 2005. A qualitative study of GPs' views of treating obesity. British Journal of General Practice 55, 750-754.

Harvey, E., Hill, A., 2001. Healthcare professionals' views of overweight people and smokers. International Journal of Obesity 25, 1253-1261.

Haslam, D., James, W., 2005. Obesity. Lancet 366, 1197-1209.

Huizinga, M.M., Bleich, S.N., Beach, M.C., Clark, M., Cooper, L.A., 2010. Disparity in physician perception of patients' adherence to medications by obesity status. Obesity 18 (10), 1932-1937.

King, D., 2011. The future challenge of obesity. Lancet 378, 743-744.

Koch, T., 1994. Establishing rigour in qualitative research: the decision trail. Journal of Advanced Nursing 19, 976-986.

Lang, A., Froelicher, E.S., 2006. Management of overweight and obesity in adults: behavioural intervention for long-term weight loss and maintenance. European Journal of Cardiovascular Nursing 5, 102-114.

Lyons, E., Coyle, A., 2007. Analysis of qualitative data in psychology. Sage, London, UK. Mariano, C., 1995. The qualitative research process. In: Talbot, L.A. (Ed.), Principles and Practice of Nursing Research. Mosby-Year Book Inc., Missouri, pp. 463-491.

Michie, S., 2007. Talking to primary care patients about weight: a study of GPs and practice nurses in the UK. Psychology, Health \& Medicine 12 (5), 521-525.

Michie, S., Hyder, N., Walia, A., West, R., 2011. Development of a taxonomy of behaviour change techniques used in individual behavioural support for smoking cessation. Addictive Behaviors 36 (4), 315-319.

Muldoon, O., McLaughlin, K., Trew, K., 2007. Adolescents' perceptions of national identification and socialisation: a grounded analysis. British Journal of Developmental Psychology 25, 579-594.

National Institute for Health and Clinical Excellence, 2006. Obesity: The prevention, identification, assessment and management of overweight and obesity in adults and children. (Clinical guidance 43) NICE, London. Retrieved from http://www. nice.org.uk/CG43.

Nawaz, H., Katz, D.L., 2001. American College of Preventive Medicine Practice policy statement. Weight management counselling of overweight adults. American Journal of Preventive Medicine 21, 73-78.

Peters, S., 2010. Qualitative research methods in mental health. Evidence-Based Mental Health 13, 35-40.

Petrich, B., 2000. Medical and nursing students' perceptions of obesity. Journal of Addictions Nursing 12, 3-16.

Poon, M.Y., Tarrant, M., 2009. Obesity: attitudes of undergraduate student nurses and registered nurses. Journal of Clinical Nursing 18 (16), 2355-2365.

Pope, C., van Royen, P., Baker, R., 2002. Qualitative methods in research on healthcare quality. Quality \& Safety in Health Care 11, 148-152.

Strauber, H., Carpenter, D., 2003. Qualitative research in nursing: advancing the humanistic imperative. Lippincott co.

Strauss, A., Corbin, J., 1998. Basics of qualitative research: techniques and procedures for developing grounded theory, 2nd ed. Sage Publications, Thousand Oaks, CA.

Taylor, C.A., Shaw, R.S., Dale, J., French, D.P., 2010. Enhancing delivery of health behaviour change interventions in primary care: a meta-synthesis of views and experiences of primary care nurses. Patient Education and Counselling 85 (2), 315-322.

The Health and Social Care Information Centre, 2011. Statistics on obesity, physical activity and diet. The Health and Social Care Information Centre, England. February.

Timmerman, G.M., Reifsnider, E., Allan, J.D., 2000. Weight management practices among primary care providers. Journal of the American Academy of Nurse Practitioners $12,113-116$.

US Department of Health and Human Services, 2001. The Surgeon General's Call to Action to prevent and Decrease Overweight and Obesity. GPO, Washington.

von Fragstein, M., Silverman, J., Cushing, A., Quilligan, S., Salisbury, H., Wiskin, C., 2008. UK consensus statement on the content of communication curricula in undergraduate medical education. Medical Education 42 (11), 1100-1107.

Wang, Y., McPherson, K., Marsh, T., Gortmaker, S.L., Brown, M., 2011. Health and economic burden of the projected obesity trends in the US and the UK. Lancet 378, $815-825$.

Watson, R., Hoogbruin, A.L., Rumeu, C., Beunza, M., Barbarin, B., Macdonald, J., et al., 2003. Differences and similarities in the perception of caring between Spanish and UK nurses. Journal of Clinical Nursing 12 (1), 85-92.

WHO, 2000. Obesity: Preventing and Managing the Global Epidemic. World Health Organization, Geneva.

WHO, 2003. World Health Report. World Health Organization, Geneva. 